\title{
Análisis de aplicaciones móviles dirigidas a la infancia: características técnicas, pedagógicas, de diseño y contenido
}

\author{
Silvia López Gómez ${ }^{1}$ (D) ; Sebastián Martín Gómez ${ }^{2}$ (D) ; María Isabel Vidal Esteve ${ }^{3}$ (C) \\ ${ }^{1}$ Universidade de Santiago de Compostela (USC), España; ${ }^{2}$ Universidad de La Laguna (ULL), España; ${ }^{3}$ Universitat de \\ València (UV), España
}

Resumen. Este artículo analiza las características técnicas, pedagógicas, de diseño y de contenido de una muestra de aplicaciones (apps) dirigidas a niñas y niños de hasta 5 años, disponibles en la plataforma Google Play. El estudio forma parte de una investigación titulada: "Los materiales didácticos digitales en la Educación Infantil. Análisis y propuestas para su uso en la escuela y el hogar» (RTI2018-093397-B-I00) en la que participan equipos de investigación pertenecientes a tres Comunidades Autónomas del Estado español (Canarias, Galicia y Valencia). La metodología utilizada se apoyó en el análisis de contenido. Se valoraron 23 apps, que destacan por ser de las más populares en Google Play durante el mes de junio de 2020, coincidentes tres de ellas con las más descargadas en los hogares españoles durante el periodo de confinamiento por la COVID-19. Para ello se aplicó una ficha de evaluación ad-hoc adaptada del proyecto Infanci@ Digit@। y se elaboraron diferentes matrices de análisis. Los resultados muestran que las apps presentan elementos curriculares de la etapa de Educación Infantil, a pesar de tener un carácter más centrado en el entretenimiento que en lo didáctico. No obstante, carecen de modelos pedagógicos activos, son poco accesibles, presentan contravalores y prima la publicidad intrusiva. Palabras clave: aplicaciones móviles; apps; materiales didácticos digitales; educación infantil; juegos digitales.

Análise de aplicações móveis destinadas a crianças: características técnicas, pedagógicas, de design e conteúdo

Resumo. Este artigo analisa as características técnicas, pedagógicas, de design e conteúdo de uma amostra de aplicativos (apps) destinados a crianças de até 5 anos de idade, disponíveis na plataforma Google Play. O estudo faz parte de uma investigação intitulada: "Materiais didáticos digitais na Educação Infantil. Análise e propostas para seu uso na escola e em casa" (RTI2018093397-B-100), na qual participam equipes de pesquisa de três Comunidades Autônomas espanholas (IIhas Canárias, Galiza e Valência). A metodologia utilizada foi baseada na análise de conteúdo. Foram avaliados vinte e três aplicativos, que se destacam como os mais populares no Google Play durante o mês de junho de 2020, três dos quais coincidem com os mais baixados nos lares espanhóis durante o período de confinamento pela COVID-19. Para este fim, foi aplicada uma ficha de avaliação ad-hoc adaptada do projeto Infânci@ Digit@l e foram elaboradas diferentes matrizes de análise. Os resultados mostram que os aplicativos (apps) apresentam elementos curriculares da fase de Educação Infantil, apesar de estarem mais focados no entretenimento do que na didática. No entanto, carecem de modelos pedagógicos ativos, são pouco acessíveis, têm contravalores e predomina a publicidade intrusiva.

Palavras-chave: aplicativos móveis; apps; materiais didáticos digitais; educação infantil; jogos digitais

Analysis of mobile applications aimed at early childhood: technical, pedagogical, design and content characteristics

Abstract. This article analyzes the technical, pedagogical, design and content characteristics of a sample of applications for children up to 5 years old, available on the Google Play platform. The study is part of an investigation titled: "Los materiales didácticos digitales en la Educación Infantil. Análisis y propuestas para su uso en la escuela y el hogar» (RTI2018-093397-B-100) in which research teams belonging to three Autonomous Communities of the Spanish State (Islas Canarias, Galicia and Valencia) participate. The methodology used was based on contentanalysis. The 23 most popular apps on Google Play in June 2020 are analyzed. Three of them are the most downloaded in Spanish homes during the period of confinement by COVID-19. For this, an ad-hoc evaluation form adapted from the Infanci@ Digit@l project was applied and different analysis matrices were prepared. The results that the applications show present curricular elements of the Early Childhood Education stage, despite having a character more focused on entertainment than on didactics. However, they lack active pedagogical models, they are not very accessible, they have counter values and intrusive advertising prevails.

Keywords: mobile applications, apps, digitalteaching materials, Early Childhood Education, digitalgames 


\section{Introducción}

Es una realidad incontestable que, desde hace ya algún tiempo, vivimos en una sociedad mediada por las tecnologías, una sociedad digital que se integra en las diferentes esferas de nuestra vida. Tanto es así, que las tecnologías cumplen actualmente una función primordial en todas las edades, y son casi un requisito para comunicarnos, relacionarnos, entretenernos e incluso para formarnos.

Siguiendo a Ditrendia (2020), a nivel mundial, el 90\% de los adultos tiene un teléfono inteligente o smarthphone, siendo el dispositivo desde donde más se accede a internet. Situándonos en el contexto español, ya en el 2013 España lideraba el puesto europeo en cuanto a implantación de smartphones, con aproximadamente 7 millones de dispositivos vendidos, de acuerdo con los datos del informe Spain Digital Future in Focus (ComScore, 2013). En dicho informe se confirmaba, además, que incluso niñas y niños de 2 y 3 años accedían con frecuencia a los smartphones y a las tabletas de sus padres, utilizando aplicaciones para jugar, dibujar, colorear o ver series infantiles de televisión.

En relación con la educación infantil, Bas y Pérez de Guzmán (2010), definen el contexto familiar como aquel en el que, tradicionalmente, niñas y niños tienen sus primeros contactos, y en el que se produce un amplio abanico de estímulos, interrogantes y asombros. En este contexto, sin embargo, estas experiencias están mediatizándose por las tecnologías (Nogueira y Ceinos, 2015) en cierto modo como consecuencia de la evolución experimentada desde el ordenador personal hacia dispositivos más prácticos y cómodos como es la tableta (Redbility, 2013). Dichas experiencias, según Cánovas et al. (2014), pueden entenderse como una oportunidad para contribuir a su educación. Además, según los resultados del estudio de Matsumoto et al. (2016), los padres entienden el acceso temprano a la tecnología de sus hijas e hijos como un proceso de familiarización y de preparación para su futuro social, escolar y laboral.

En esta misma línea, según autores como Asorey y Gil Alejandre (2009) o Ebner, Schönhart y Schön (2014), la presencia de la tableta se observa principalmente en los hogares, sin embargo, también está constatándose un uso didáctico incluso en las escuelas, dentro de lo que se denomina $M$ Learning (Madrid, Mayorga y Núñez, 2013), o lo que es lo mismo, aprendizaje mediado por dispositivos móviles. Lo que estos datos nos permiten cotejar, es que la penetración de las tecnologías en los espacios de uso más frecuente para ellos, como es el hogar o los centros educativos, está posibilitando que niñas y niños se conviertan en usuarios de estas tecnologías, lo que garantiza que se suban al carro de la revolución tecnológica (Pascual, 2019). 
Así pues, por suerte o por desgracia, tal y como afirma Sanchís (2019) "encontrar a un niño o niña de pequeña edad enganchado a una pantalla es algo habitual en estos tiempos" (p. 722). Podemos verlos jugando con el móvil o la tableta de algún familiar y, pese a que existen numerosas apps pedagógicas que se implementan como herramientas de apoyo a la educación (López-Raventós, 2016; Marín y Martín, 2014), en demasiadas ocasiones, los más pequeños las descargan y las utilizan sin ningún criterio de selección ni control parental. Pero, ¿qué aplicaciones se descargan?, ¿qué características reúnen?, ¿qué elementos las componen?

Para contribuir a dar respuesta a estas cuestiones, en el presente estudio se ha realizado un análisis interpretativo de una muestra de aplicaciones (apps) dirigidas a niñas y niños de hasta 5 años, disponibles entre las más populares en la plataforma Google Play.

Asimismo, se procura alcanzar los siguientes objetivos específicos:

- Analizar las características de las aplicaciones desde el punto de vista tecnológico y de diseño.

- Conocer los rasgos pedagógicos, de evaluación y de seguimiento de las aplicaciones.

- Identificar las singularidades de las aplicaciones atendiendo a su contenido.

Conviene recordar que este artículo deriva de una investigación en desarrollo titulada: "Los materiales didácticos digitales en la Educación Infantil. Análisis y propuestas para su uso en la escuela y el hogar" (Acrónimo: Infanci@ Digit@I), la cual pretende analizar las características pedagógicas y técnicas de los materiales didácticos digitales (MDD) dirigidos a infantes con edades comprendidas entre los 3 y 6 años, así como estudiar cómo se utilizan estos recursos tanto en las familias como en las aulas de segundo ciclo de Educación Infantil.

La investigación Infanci@ Digit@I aglutina a su vez cuatro estudios diferentes pero complementarios entre sí, llevados a cabo de forma paralela por grupos de investigación pertenecientes a las Comunidades Autónomas de Canarias, Galicia y Valencia. De forma resumida los estudios son los siguientes:

- Estudio 1: Análisis de recursos digitales destinados a segundo ciclo de Educación Infantil (plataformas, apps, materiales didácticos digitales). 
- Estudio 2: Identificación de las opiniones y visiones que el profesorado de este ciclo y las familias tienen sobre los materiales digitales.

- Estudio 3: Realización de diferentes estudios de casos para conocer cómo se utilizan esos recursos.

- Estudio 4: Elaboración de una guía dirigida al profesorado y a las familias con recomendaciones de buenas prácticas para el uso de recursos digitales.

Con el desarrollo de este trabajo también se desea concienciar al profesorado, a las familias y a las instituciones sobre la importancia de saber seleccionar, usar y elaborar contenidos digitales para la infancia.

\section{Método y materiales \\ 2.1 Metodología}

Con el fin de analizar las características de las apps seleccionadas, el planteamiento metodológico empleado es el análisis del contenido. Este se entiende como el conjunto de técnicas de investigación cuyo objetivo es la obtención del discurso que subyace a la producción documental de una determinada realidad social. Tal y como apunta Krippendorff (1990), la puesta en práctica habitual de estas técnicas consiste en la realización de inferencias aplicables a un contexto a partir de ciertos datos. Se trata, como indica Bardin (1986), de la búsqueda de indicadores cualitativos o cuantitativos por procedimientos descriptivos, sistemáticos y objetivos, en el contenido de los mensajes establecidos en un determinado contexto social. La obtención de estos indicadores permitirá la inferencia de conocimiento sobre la producción y recepción de dichos mensajes.

Aunque el análisis de contenido suele asociarse más a la evaluación de libros de texto, esta metodología se utiliza también en otros contextos de investigación con distintas finalidades. Uno de estos contextos es el análisis del software, en el que también se puede incluir el análisis de las aplicaciones móviles (López-Gómez, 2018), que es lo que se pretende realizar en la presente investigación.

Tal y como advierte Zapico (2012), el análisis de contenido puede ser descriptivo o inferencial. El análisis descriptivo tiene como objetivo la obtención de una descripción de la forma y el contenido de los mensajes, que posibilite la realización de generalizaciones mediante la asociación con otros recursos. Por su parte, en el análisis inferencial, el objetivo es el plan- 
teamiento de cuestiones relacionadas con el contenido que van más allá de su mera descripción. De esta manera, la investigación adquiere un carácter explicativo.

Esta investigación se acoge a los siguientes presupuestos del enfoque interpretativo en ciencias sociales, siguiendo y coincidiendo con Rodríguez-López (1997):

- Desde este estudio no se pretenden generalizar los resultados conseguidos a todas las apps dirigidas a la infancia en Google Play, sino presentar una aproximación fundamentada sobre el diseño técnico, pedagógico y de contenido de las aplicaciones.

- En este trabajo no se ofrecerá una serie de patrones cerrados y generalizables. Se pretende identificar ciertos trazos comunes entre las apps, pero sería erróneo reducir todos los elementos, percepciones y representaciones de la realidad que en ellas se proporcionan a un conjunto de categorías inflexibles y rígidas. Lo que se desea, es realizar descripciones de algunos de los elementos que las conforman y de cómo se presentan, para elaborar categorías de análisis que hagan posible una fundamentada y reflexiva interpretación de dichos elementos.

- En el análisis de los resultados se pondrán en práctica, tanto procedimientos inductivos como deductivos a partir de la información captada mediante el uso de un instrumento de análisis que será presentado más adelante.

Por tanto, y en virtud de lo expuesto, la investigación se acogería a algunos de los principios básicos del paradigma interpretativo, compaginando la descripción y análisis de las aplicaciones más populares de Google Play dirigidas a niñas y niños de hasta 5 años, con la interpretación de sus elementos.

\subsection{Muestra}

La muestra del estudio la componen un total de 23 aplicaciones disponibles en la plataforma de distribución digital Google Play. Las apps o aplicaciones son una tipología de software desarrollado principalmente para dispositivos móviles, tales como las tabletas y los teléfonos inteligentes. En la actualidad, existe una gran diversidad de aplicaciones, como la creación de vídeos, edición de fotografías, acceso a guías de viajes, a noticias, lectura de libros, etc. y para pasar ratos de entretenimiento y juego. 
En este estudio se ha optado por seleccionar apps dirigidas a niñas y niños de hasta 5 años. Los criterios seguidos para la selección de la muestra de estudio han sido los siguientes:

- La selección de las aplicaciones se realiza entre el 25 y 28 de junio de 2020.

- Se opta por elegir las aplicaciones de entretenimiento incluidas en la categoría "Familia" dentro de Google Play, subcategoría "Hasta 5 años".

- Se decide que las aplicaciones deben ser las "Más populares" y dentro de estas que formen parte del "Top aplicaciones".

luación:

La siguiente tabla recoge las 23 apps seleccionadas para su eva-

Tabla 1. Aplicaciones seleccionadas para el análisis.

\begin{tabular}{ll}
\hline \multicolumn{2}{c}{ Muestra de aplicaciones } \\
\hline - LEGO® Tower & - Pop Globos Juego para niños \\
- PlayKids - Series, Libros y Juegos Educativos & - Niños Aprenden Profesiones \\
- ¡Comida Divertida 2! Juegos & - Juego Educativo Niños 5 \\
- Sonic Dash & - Minion Rush: Gru - Mi Villano Favorito \\
- Salón de uñas Hello Kitty & - MY LITTLE PONY: mágico \\
- Disney Magic Kingdoms: ¡Crea Tu Parque Mágico! & - Buenas noches, Caillou \\
- PJ Masks: Moonlight Heroes & - Farming Simulator 14 \\
- Peppa Pig: Paintbox & - Masha y el oso - Juegos educativos \\
- Juegos Infantiles Educativos & - Hot Wheels: Race Off \\
- La consulta de Caillou & - Baby panda supermarket \\
- YouTube Kids & Clan RTVE \\
- Boing App - Tus series de dibujos y juegos gratis &
\end{tabular}

Fuente: Elaboración propia.

\subsection{Instrumento de análisis y proceso del estudio}

Para el análisis de las aplicaciones se utilizó una ficha de evaluación ad-hoc adaptada del proyecto Infanci@ Digit@I. Este instrumento fue elaborado en un proyecto anterior en el que participaron los mismos equipos de investigación, titulado: "La escuela de la sociedad digital: análisis y propuestas para la producción y uso de los contenidos digitales educativos" (Acrónimo: Escuel@ Digit@I. EDU2015-64593-R) 
El instrumento ha sido validado y sometido a juicio críticos de expertos externos, en concreto por ocho personas especialistas en materiales didácticos procedentes tanto del ámbito nacional como internacional. Consta de los siguientes grandes apartados: Datos del evaluador/a; Identificación del material o recurso educativo; Estructura del material; Dimensión tecnológica; Dimensión de diseño; Dimensión Pedagógica; Dimensión de contenido; Evaluación y seguimiento; Comentarios finales.

Para llevar a cabo este estudio, en primer lugar se cumplimentaron las fichas de evaluación de todas las aplicaciones seleccionadas y, posteriormente, se elaboraron ad-hoc diferentes matrices de análisis, siguiendo las principales categorías y subcategorías del instrumento, para finalmente proceder con el análisis de las características técnicas, pedagógicas, de diseño y contenido de las aplicaciones.

Siguiendo a Miles, Huberman y Saldaña (2014) las matrices de análisis se caracterizan por ser "esencialmente la intersección de dos listas, configuradas como filas y columnas" (p. 109), que tienen como finalidad facilitar el estudio de una serie de variables dependientes a través de la interrelación y agrupamiento de las respuestas proporcionadas por un grupo de sujetos. Poseen un marcado carácter visual, ya que permiten concentrar numerosa información en una única tabla o figura. Según los mismos autores, el formato matricial también posibilita añadir conclusiones o comentarios inferenciales de la persona que investiga.

\section{Resultados}

A continuación, se presentan los resultados obtenidos a partir del análisis de contenido y que se dividen en las grandes dimensiones en las que se articula el estudio.

\subsection{Identificación del material o recurso educativo}

La primera dimensión es la referida a los datos de identificación de las aplicaciones, en la que se recogen aspectos primordiales que caracterizan estos recursos, tales como título, autoría, idiomas, tipo de licencia, principales destinatarios, entre otras.

En este bloque se constata que la mayoría de las aplicaciones que componen la muestra, un $48 \%$ del total, han sido subidas a Google Play en la categoría de educación. Las restantes se distribuyen entre aplicaciones 
pertenecientes al género de acción y aventura (26\%), juegos simulados (13\%) y aplicaciones de música y vídeo (13\%). Estas últimas coinciden con tres apps que se encuentran entre las más descargadas durante el periodo de confinamiento en los hogares españoles debido a la Covid-19 entre los meses de marzo y junio de 2020. En concreto fueron: YouTube Kids, Boing App y Clan RTVE. Las tres aplicaciones se basan en contenido multimedia, destacan los contenidos en vídeo. Tanto Boing App como Clan RTVE provienen de canales de televisión que cuentan con una larga trayectoria en la distribución y producción de contenido televisivo para niños/as de distintas edades: series de televisión, dibujos animados comerciales, etc. YouTube Kids es la versión adaptada al contenido infantil de la popular app y red social de Google. Estas aplicaciones están diseñadas para el uso en el núcleo de la familia.

Por otra parte, el $100 \%$ de estas apps se clasifican con el código PEGI 3. El código PEGI (Pan-European Game Information) es un sistema de clasificación por edades desarrollado por la Federación de Software Interactivo de Europa (ISFE) con el objeto de ayudar a los progenitores a tomar decisiones informadas a la hora de adquirir un contenido de entretenimiento, sobre todo videojuegos, pero también aplicaciones móviles, películas, etc. Esta clasificación por edades $(3,7,12,16$ y 18) no tiene en cuenta el nivel de dificultad ni las habilidades necesarias para utilizar la aplicación, pero sí indican la idoneidad de su contenido. En este caso, las aplicaciones objeto de análisis deberían estar libres de sonidos e imágenes que asustan a las/os niños, ni podrían incluir un lenguaje soez.

En cuanto a la autoría de las mismas, en la muestra se encuentran cuatro aplicaciones desarrolladas en el contexto español, dos de ellas por la empresa bilbaína AppQuiz (Juegos Infantiles Educativos; Masha y el oso - Juegos educativos), una por pesCAPPs de Castellón (Juego Educativo Niños 5) y otra de la RTVE Medios Interactivos (Clan RTVE). El origen de las restantes es muy diverso, el 13\% fueron desarrolladas desde Francia por parte de Gameloft, otro 13\% desde Canadá por Budge Studios, pero también se encuentran aplicaciones, en menor porcentaje, elaboradas desde California, Israel, Brasil, Polonia, Suecia, Suiza, China o Londres, tanto por parte de pequeños estudios como por grandes desarrolladoras de la industria, como puede ser SEGA.

Los idiomas también resultan variados, sin tener en cuenta la app YouTube Kids (disponible en más de 80 países), se han contabilizado 38 idiomas, estando el inglés presente en el $100 \%$ de las aplicaciones a través de textos, audios u opciones dentro de la app. Le sigue el español (95\%), chino (73\%, aunando chino simplificado y chino tradicional), francés (64\%), portugués (59\%), alemán e italiano (ambos $50 \%$ ) y ruso (45\%). En menor 
medida el japonés (36\%), el coreano y el turco (con un $32 \%$ cada uno) y también otros idiomas tan diversos como el polaco, el danés, el malayo, el neerlandés, etc.

Gran parte de las aplicaciones han sido actualizadas durante el año 2020 (el 78\%), la más antigua es Peppa Pig Paintbox, con fecha del día 3 de octubre de 2016.

Las aplicaciones más descargadas, con más de 100.000.000 instalaciones, pertenecen a los juegos del género de acción y aventuras (Minion Rush y Sonic Dash), pero también se incluye en este ránking el juego de simulación Salón de uñas Hello Kitty y la aplicación YouTube Kids. El 35\% de las apps han sido descargadas más de 50.000.000 veces.

En general, el tipo de licencia que ofrecen es gratuita, pero siguen lo que se denomina planteamiento free to play, es decir permiten ser utilizadas o jugadas de forma gratuita en su versión básica a la vez que abren opciones de comercialización, como por ejemplo mediante la adquisición de elementos que faciliten el progreso en la app o el acceso a contenido adicional. Las compras incluidas dentro de las aplicaciones de la muestra objeto de estudio se encuentran comprendidas entre los 0,50 y los 109,99 por artículo. Únicamente un $26 \%$ no incluyen productos para comprar dentro de la aplicación de forma directa, entre estas se encuentran las relacionadas en la categoría entretenimiento (música y vídeo): YouTube Kids, Clan RTVE y Boing App.

\subsection{Dimensión tecnológica}

A través de esta dimensión de análisis se pretende identificar las características tecnológicas más destacables de las aplicaciones.

En general, la navegabilidad de las apps se considera buena, sencilla e intuitiva, por varios motivos: los accesos son táctiles, cuentan con pocos botones u opciones y emplean mayoritariamente un lenguaje icónico o visual. Si bien, en las versiones gratuitas de algunas de ellas esta navegabilidad presenta problemas debido a los anuncios invasivos que saltan a la pantalla, como en las apps Juego Educativo Niños 5, ¡Comida divertida 2!, My Little Pony: mágico, entre otras. En relación con este aspecto, es destacable la aplicación Buenas noches, Caillou en las que las opciones de pago se protegen con un código numérico o "captcha parental". 
Todas las aplicaciones son multiplataforma disponibles para tabletas y teléfonos inteligentes. En general, tanto para sistemas operativos Android como iOS. Además, podrían ejecutarse en ordenadores si se utiliza un emulador como BlueStacks App Player.

La velocidad de carga se evalúa como alta, no obstante, las cargas iniciales pueden ser moderadas o algo lentas si no se cuenta con una buena conexión a la red. A mayor tamaño de la app, mayor suele ser el tiempo de descarga, y en la muestra se encuentran tamaños que van desde los $122 \mathrm{M}$ (LEGO® Tower) hasta los 13M de Juegos Infantiles Educativos, pero este tamaño también puede variar según el dispositivo.

Respecto a la accesibilidad, entre la muestra se encuentran aplicaciones que presentan opciones de configuración, como Pop Globos Juego para niños, en la que se puede adecuar el tamaño y la velocidad de desplazamiento de los objetos, y permite la activación/desactivación de sonidos. Pero en general, no se consideran accesibles, ni se tiene en cuenta el acceso de personas con diversidad funcional.

\subsection{Dimensión de diseño}

En esta dimensión se pretenden recoger las características de diseño y de funcionalidad más destacables de las aplicaciones.

En general, salvo Juego Educativo Niños 5, las aplicaciones cuentan con un diseño atractivo o muy atractivo, en las que destacan las imágenes coloridas, la música alegre, simpáticas animaciones y elementos interactivos. Mayoritariamente las ilustraciones se adaptan al nivel psicoevolutivo del principal grupo destinatario, muchas de las cuales integran personajes de dibujos animados conocidos por las niñas y niños. Además, prima la representación visual sobre el texto escrito, aunque en Farming Simulator 14 se integra demasiado texto y es muy complejo para menores de 8 años.

En la muestra también se encuentran aplicaciones con estereotipos sexistas, es el caso de la app Salón de uñas Hello Kitty, en la que todas las manos que aparecen presentan rasgos que se suelen asociar al género femenino y en la que predomina el color rosa.

\subsection{Dimensión pedagógica}

En cuanto al análisis de la dimensión pedagógica de las distintas apps, tal y como queda reflejado en la metodología, se ha tenido en cuenta la finalidad de la app, sus características más destacables, su adaptabilidad 
a los distintos ritmos de aprendizaje o la tipología de actividades que contiene, entre otros. Así pues, tras aglutinar la información obtenida en matrices, encontramos los siguientes resultados.

Por una parte, la mayoría de las aplicaciones destacan por su finalidad lúdica (Salón de uñas Hello Kitty, My Little Pony: mágico o PJ Masks: Moonlight Heroes), sin embargo, otras como Niños Aprenden Profesiones, Kids Balloon Pop Games, Buenas noches Caillou, Clan RTVE o Peppa Pig Paintbox, podría decirse que también contienen una finalidad parcialmente educativa. De entre las que podrían clasificarse como educativas en cierta medida, el modelo pedagógico que subyace es mayormente conductista, con algunas pinceladas del modelo naturalista o experiencial, a partir actividades de ensayo y error, por ejemplo. En esta misma línea, el tipo de actividades que destacan son de creación y de reproducción de modelos acompañadas siempre del trabajo de la coordinación óculo-manual. También se detectan actividades de descubrimiento o iniciación, de aplicación y refuerzo, de toma de decisiones, de motricidad fina o de discriminación, de concentración y, por supuesto, de diversión.

En cuanto a las características más destacables de cada una de ellas, podemos decir que estas son múltiples y diversas, aun así, se han identificado aspectos comunes como, por ejemplo, que muchas de ellas requieren de pagos, -como ya se ha comentado-, o que la mayoría de ellas no se vinculan al currículum de educación infantil; y algunas, incluso ni se ajustan al público objetivo, ya que contienen instrucciones en texto, difíciles de comprender para niños de 3-5 años. Como se ha mencionado, son muy diversas; algunas son aplicaciones de simulación: de conducción (Hot Wheels Race Off), de construcción (LEGOß Tower), de compra (Baby Panda Supermarket), de cuidar de una granja (Farming Simulator 14) o de reproducción de música y vídeos (YouTube Kids, Boing App). Y otras; por su parte, contienen secuencias de actividades o minijuegos, para ir desarrollando paulatinamente (como La consulta de Caillou). Además, ninguna de ellas es editable o modificable, más allá de valores técnicos como el volumen, el idioma o la vibración.

Por otra parte, es de destacar que ninguna de las aplicaciones analizadas explícita los objetivos ni tampoco los contenidos de aprendizaje, del mismo modo que ninguna contiene actividades para diferentes ritmos de aprendizaje, con las excepciones parciales de Juegos Infantiles Educativos que sí contiene algunas actividades más complejas que otras, pero que no permite al niño o niña elegir con cuál desea jugar; y, de YouTube Kids y Clan RTVE que -al iniciar sesión-solicitan al padre, madre o tutor que marque la categoría en la que se ubica el niño/a, para seleccionar los contenidos que se mostrarán (niños preescolares: hasta 4 años, niños pequeños: 5-7 
y niños mayores 8-12 en YouTube Kids; y, Preescolar, Infantil y Junior en Clan RTVE). Esto se relaciona, de alguna forma, con la planificación del aprendizaje y es que, ninguna de las aplicaciones analizadas lo promueve, únicamente, en algunas ocasiones puede trabajarse la gestión de recursos, si existe cierta actitud de guía por parte de algún adulto que acompañe al niño/a en el proceso.

En cuanto al número de jugadores implicados, la mayoría son de uso individual, a excepción de Farming Simulator 14 que permite la opción multijugador. Por lo que también, este hecho, supone que no favorecen el trabajo cooperativo, ni la implicación de la familia, ni la interacción con el entorno, ni con los compañeros ni con los adultos, a excepción de algunas como My Little Pony o Disney Magic Kingdoms, que podrían necesitar de la intervención del adulto para la completa comprensión de las instrucciones ofrecidas, o YouTube Kids para activar el control parental al inicio.

Asimismo, algunas de las aplicaciones analizadas se ubican con contenidos alejados del entorno de los niños y niñas; en otras, su relación surge de la aparición de unos personajes de televisión que pueden ser más o menos conocidos por el público objetivo (como Minion Rush, Sonic Dash, Salón de uñas Hello Kitty, My Little Pony: mágico, Clan RTVE o Disney Magic Kingdoms); y, en otras, sí están más relacionadas con el imaginario de los niños y niñas, en las que, por ejemplo, aparecen objetos cotidianos y sociales cercanos a estos, como los juguetes, los alimentos, las prendas de ropa, la rutina del sueño, el momento de ir al baño, la visita al médico o al supermercado.

Finalmente, en lo referente a la presencia de temas transversales, la mayoría destaca por no trabajarlos, sin embargo, sí que aparecen en alguna de ellas temas como la creatividad, las emociones, la educación en valores o la vida saludable ( $L E G O \Theta$ Tower, Peppa Pig: Paintbox, Clan RTVE, La consulta de Caillou, Baby panda supermarket). También, algunas de las apps analizadas, puede considerarse que contribuyen al desarrollo de competencias, como la competencia para la autonomía e iniciativa personal; la competencia matemática; la competencia en el conocimiento y la interacción con el mundo físico; la competencia en comunicación lingüística; la competencia de aprender a aprender; y, la competencia digital y el tratamiento de la información.

\subsection{Dimensión de contenido}

Centrando la atención en la dimensión de contenido, se presentan los rasgos más característicos atendiendo a una serie de categorías de interés: la posibilidad de seleccionar el contenido y actividades en función de 
los intereses del alumnado y de los diferentes ritmos de aprendizaje; cómo los contenidos contribuyen al conocimiento de la diversidad socio-cultural y política; el favorecimiento de un tratamiento diferenciado en función del contexto social, cultural y ambiental de aplicación; la incorporación en estas aplicaciones de personajes identificados como estudiantes con edades 0 con características similares a los potenciales destinatarios/as; la tipología de personajes que aparecen en los materiales (como animales, personas, personajes comerciales conocidos, etc.); si el contenido icónico y textual refleja la diversidad funcional, sexual, cultural, etc.; si el material responde a las demandas curriculares de la Educación Infantil; la lógica que organiza y secuencia el contenido; si se abordan ámbitos del saber o conocimiento de distinta naturaleza; y, por último, si las actividades o procesos contemplados en estas aplicaciones se pueden resolver de forma manipulativa con recursos del aula o en el entorno cercano como es el hogar.

Tras Ilevar a cabo el proceso de análisis conjunto mediante la creación de matrices, se presentan los siguientes resultados exponiendo aquellos aspectos más relevantes de cara a la investigación.

En cuanto a la posibilidad de seleccionar el contenido y actividades planteadas en las apps en función de los intereses del alumnado y de los diferentes ritmos de aprendizaje, aproximadamente la mitad de los recursos analizados permiten escoger algún aspecto, aunque no siempre referidos al nivel de complejidad o ritmo de aprendizaje. Destaca la posibilidad de escoger entre diferentes pantallas o escenarios de juego, e incluso seleccionar diferentes micro juegos dentro de la misma aplicación. En estos casos se podría realizar la elección en función de los intereses del público objetivo, pero no se tendrían en cuenta otros factores. En otros casos, únicamente se permite escoger el avatar, como ocurre en el juego Peppa Pig Paintbox, donde se da a elegir entre dos personajes para comenzar. Destaca de forma significativa que solo haya una app que permite escoger los procesos o actividades planteadas en función de la etapa educativa: Masha y el oso - Juegos educativos.

Ninguna de las aplicaciones contempladas en el análisis cuenta con contenidos que favorezcan el conocimiento de la diversidad sociocultural y política. De hecho, en varias ocasiones este aspecto viene dado por el conocimiento previo de los personajes comerciales en los que se basa el juego: dibujos animados de series de televisión. Se pueden observar algunos contenidos que, de forma indirecta, contribuyan a aprendizajes relacionados con el conocimiento del medio cercano, pero no abordan la diversidad social o cultural de forma específica. De forma similar ocurre con las aportaciones que propician un trato diferenciado en función del contexto social, cultural 
o ambiental; las cuales son inexistentes. Esto puede deberse principalmente a la naturaleza de las apps y juegos; y, sobre todo, a las nociones en estos temas que tiene el público objetivo: niñas y niños de Educación Infantil.

En cuanto a la diversidad sexual o de etnia, este es un aspecto poco presente en las narrativas u opciones de juego. Sin embargo, en algunas como es el caso de Peppa Pig Paintbox, se permite escoger entre un avatar masculino o uno femenino al comienzo del juego. Llama la atención en este caso, además, que se potencia un estereotipo de género, usando una relación de colores azules cuando se escoge el personaje masculino (George), cambiando a tonos rosas cuando se usa el personaje femenino (Peppa).

La mayor parte de los juegos analizados no cuentan con personajes que guíen el recorrido por el juego, o avatares con características similares a los usuarios a las que van destinadas estas apps. Muchas de ellas no incluyen esta figura por la propia naturaleza del juego, pero integran personajes comerciales derivados de contextos narrativos externos: series de televisión de dibujos animados. Un ejemplo de esto son los juegos La consulta de Caillou - Masha y el oso - Juegos educativos. Este aspecto está relacionado con la tipología de personajes que aparecen en estas aplicaciones. Es frecuente encontrar personas (adultos y niños/as) y elementos y objetos animados como protagonistas del juego. Aunque lo más popular es encontrar animales como personaje principal en la narrativa.

La relación con el currículo asociado a la etapa de la Educación Infantil es un aspecto que sí está presente de forma positiva. Es común encontrar actividades y procesos en estas apps o juegos que trabajan contenidos curriculares relacionados con los números, el abecedario, los colores y formas básicas. Otras, en cambio, están enfocadas a desarrollar la psicomotricidad fina o la lateralidad. En menor medida, se encuentran casos, como el de Juego educativo niños 5 / Kids games 5 , en los que se presentan propuestas para abordar las emociones, contribuyendo a saber cómo identificarlas. Hay que enfatizar que en todas estas aplicaciones el lenguaje artístico está muy presente. De igual forma, los ámbitos del saber, saber hacer; y, saber estar o ser también están presentes.

Por otra parte, también se hallan apps que no contemplan el currículo. Estas son apps mucho más comerciales, que centran la atención en la vertiente lúdica y del entretenimiento, sin ahondar en aprendizajes propios de la etapa. Se identifican casos, incluso, que se alejan bastante del público objetivo en cuanto a los niveles de dificultad, como es el caso de Baby panda supermarket, el cual indica que está dirigido a alumnado de hasta 8 años. 
Otro elemento a tener en cuenta, en este análisis del contenido, ha sido la secuenciación lógica del itinerario de actividades y propuestas dentro de los juegos. La mayoría están organizadas en niveles de dificultad ascendentes, aunque también se encuentran casos en los que las pantallas o escenarios de dificultad es aleatorio. En general, no se tienen en cuenta los ritmos de aprendizaje.

Por último, es importante abordar el hecho de que todas las aplicaciones de este análisis no contemplan una parte de desarrollo manipulativo; pues, todas son juegos ideados para dispositivos móviles. Sin embargo, hay algunos contenidos planteados de forma virtual que tienen una fácil aplicación o adaptación al contexto real. Por ejemplo, el juego $L E G O \circledast$ Tower, basado en construcciones con bloques y piezas.

\subsection{Evaluación y seguimiento}

Ninguna de las aplicaciones detalla criterios o estrategias de evaluación, incluso las consideradas educativas, las cuales tampoco ofrecen la posibilidad de obtener un informe de evaluación acerca de las actividades o retos llevado a cabo. No obstante, dado que muchas de las aplicaciones tienen un marcado componente lúdico, esta evaluación podría medirse en función del avance o éxito que se tenga en la aplicación, por ejemplo, en PJ Masks: Moonlight Heroes tras la superación de cada pantalla se reporta el nivel de éxito y se obtienen recompensas (puntos, desbloqueo de niveles, feedbacks positivos sonoros o visuales, ...). Sin embargo, esta supuesta evaluación positiva puede resultar engañosa, ya que existen aplicaciones que incitan a recurrir a micropagos para superar situaciones complejas y dar sensación de avance, es el caso de la app Disney Magic Kingdoms: crea tu propio parque mágico.

\section{Conclusiones y discusión}

Entre los dispositivos tecnológicos disponibles, los dispositivos móviles pueden resultar interesantes y útiles para el desarrollo cognitivo en la infancia, ya que facilita el aprendizaje a través de estímulos visuales, sonoros, de dibujo, etc., en un entorno sencillo y atrayente; y, puede llegar a fomentar la autonomía, la creatividad, la motivación e incluso la adquisición y desarrollo de ciertas competencias, consideradas, hoy en día, como básicas e imprescindibles (Nogueira y Ceinos, 2015). Algunos autores como Romero et al. (2012) no dudan de su potencial, llegando incluso a plantearse 
su uso como un medio más para la adquisición, lo más pronto posible, de competencias digitales necesarias para un buen desempeño en la Sociedad de la Información.

Sin embargo, resulta esencial en este punto, que las familias asuman un rol de compromiso con la intención de regular el uso de tecnologías de este tipo por parte de sus hijos e hijas, velando por su seguridad y protección (Quiñones, 2014), ya que como afirma Herodotou (2018), es fundamental crear un ecosistema digital seguro y relevante para niñas/os que garantice experiencias innovadoras y entretenidas. En este punto, la escuela juega un papel importante mediante la acción orientadora de maestros y maestras, así como de otros especialistas (técnicos de educación infantil, orientadores/ as escolares, etc.) que puedan interactuar con las familias, colaborando y ofreciendo información y pautas para realizar un uso correcto de dichas herramientas, con el fin de evitar y/o prevenir posibles problemas de conducta, que puedan derivar en trastornos del desarrollo en la infancia (Bas y Pérez de Guzmán, 2010).

A continuación, se presentan las conclusiones de carácter general obtenidas en esta investigación, con aquellos aspectos a destacar, tanto positivos como a mejorar, que constituyen una síntesis de las fortalezas y debilidades de las aplicaciones analizadas destinadas a la etapa de Educación Infantil.

En primer lugar, se destaca positivamente que muchas de las apps permiten la adquisición de rutinas que son necesarias desarrollar en edades tempranas, como llevar una buena alimentación, ir al médico o tener una correcta higiene. Además, en ciertas ocasiones, también se encuentran actividades o juegos que permiten la adquisición de hábitos de atención, observación, exploración, análisis, comprensión, razonamiento, expresión oral, memoria, orientación, etc. Muchas de las aplicaciones trabajan de forma directa e indirectamente la motricidad fina, haciendo que el niño o niña que juegue, por ejemplo, lleve a cabo tareas de dibujo. Estas conclusiones son similares a las obtenidas en otros estudios (González et al., 2007; González y Gutiérrez, 2014), en los que se afirma que el uso de apps o juegos digitales ofrecen mejoras en la coordinación de los más pequeños y, además, los hace persistentes en la consecución de tareas, ya que se focalizan en un objetivo concreto: superar un nivel o terminar un juego, por ejemplo.

También se extrae positivamente, que los elementos curriculares de la etapa de Educación Infantil están presentes. Se pueden encontrar contenidos relacionados con diferentes áreas, ámbitos y saberes. Destacan sobre todo los relacionados con el conocimiento de sí mismo y autonomía personal; y, el conocimiento del entorno. También se trabajan conocimientos básicos, como conocer e identificar colores, letras, números, formas básicas, 
etc. La adecuación del contenido a la edad y a los conocimientos previos del público objetivo se considera, según algunos autores, como Crescenzi, Valente y Suárez (2019), como una de las principales características de los recursos digitales, apps en este caso, que contribuyen a definir la protección digital desde una perspectiva ética y crítica.

A nivel de desarrollo, las aplicaciones destacan positivamente por presentar imágenes y recursos visuales de calidad. Muchas de ellas están diseñadas en base a personajes y escenarios comerciales conocidos tanto por los niños/as como por las familias. En general, se presentan apps fáciles de usar con una interfaz intuitiva con animaciones fluidas, buenos tiempos de respuesta y estable a nivel de funcionamiento. Algunas de ellas cuentan con opciones útiles para padres y madres como puede ser el control parental o el bloqueo de ciertas opciones.

También se puede entender como positivo el hecho de que todas las apps presentadas son gratuitas, aunque algunas de ellas cuentan con compras in-app, y que estén disponibles en diferentes idiomas, lo que puede favorecer, en gran medida, la competencia lingüística (Del Moral, 2004; Rico y Aguado, 2016).

Por otra parte, como aspectos negativos, hay que enfatizar que la mayoría de las aplicaciones no pueden considerarse material didáctico digital. Entendiendo por material didáctico digital, aquel formado por un conjunto de distintos objetos digitales para generar una experiencia de enseñanza y aprendizaje (Area, 2017). Aunque las apps objeto de estudio cuenten con aprendizajes implícitos, la mayoría de ellas tienen un carácter más centrado en el entretenimiento que en lo didáctico. Tienen una estructura unidireccional destinada a recorrer y superar niveles de diferentes grados de dificultad, mediante un modelo de ensayo-error, sin plantear otras posibilidades didácticas. Igualmente, tampoco se plantean aprendizajes con modelos pedagógicos activos, participativos o colaborativos. Es por ello, que la figura de las familias o del docente no tiene cabida en estos recursos.

Por otro lado, en muchas ocasiones se encuentran aspectos negativos a nivel contenido como puede ser la aparición de contravalores como el sexismo o la violencia, aspectos que vienen dados por la mecánica y temática de los juegos. Del mismo modo, y de forma muy similar a lo obtenido en el estudio de Crescenzi y Grané (2016), se hallan procesos de dificultad elevada que se alejan del público objetivo de estas aplicaciones, ya que suelen contener mensajes escritos vinculados con informaciones relevantes, instrucciones o feedback. Y es que, tal y como apunta Guernsey (2013), pese a que el mercado está saturado de apps para niñas y niños, la etiqueta de «educativa» o «infantil» no indica que estas apps hayan sido validadas 
ni testeadas para ser clasificadas como tal. Muestra de ello es que, como es habitual, las aplicaciones gratuitas están diseñadas para ganar dinero por medio de la publicidad o a través de compras in-app o micro pagos, y en algunos casos la publicidad Ilega a ser intrusiva, mostrando incluso anuncios destinados a un público adulto.

A nivel técnico, en todos los casos se presentan aplicaciones diseñadas para captar la atención y llevar a cabo un uso prolongado de la aplicación. Es frecuente que no haya un final como tal o una meta a la que llegar, por lo que el usuario puede estar largos ratos jugando. Esto conlleva graves daños, desde el cansancio de la vista hasta la adicción a la tecnología, pasando por el incremento de los problemas atencionales, tal y como apuntan Moreira y Gómez (2019). Los resultados en cuanto accesibilidad coinciden con la investigación de López-Gómez (2018), en donde se concluye que la accesibilidad en las aplicaciones y videojuegos, sean didácticos o comerciales, es una tarea pendiente, lo que contribuye a la exclusión de los colectivos afectados (Mangiron, 2011).

Coincidimos plenamente con los autores Crescenzi, Valente y Suárez (2019) cuando indican que: "proteger al menor desde una perspectiva ética e inclusiva implica promocionar la formación crítica del alumnado desde la escuela infantil, para su integración en el mundo digital" (p. 101).

\section{Agradecimientos}

Esta contribución forma parte del proyecto "Los materiales didácticos digitales en la Educación Infantil. Análisis y propuestas para su uso en la escuela y el hogar" (Infanci@ Digit@I: RTI2018-093397-B-I00) del Programa Estatal de I+D+i Orientado a los Retos de la Sociedad; del proyecto de tesis doctoral con referencia FPU17/00372 financiado por el Ministerio de Ciencia, Innovación y Universidades; y del proyecto de tesis doctoral con referencia TESIS2018010043 financiado por Agencia Canaria de Investigación, Innovación, y Sociedad de la Información.

\section{Referencias}

Area, M. (2017). La metamorfosis digital del material didáctico tras el paréntesis Gutenberg. RELATEC, 16(2), 13-29. https://doi.org/10.17398/1695-288X.16.2.13

Asorey, E. y Gil Alejandre, J. (2009). El placer de usar las TIC en el aula de Infantil. CEE Participación Educativa, 12, 110-119. 
Bardin, L. (1986). El análisis de contenido. Madrid: Akal.

Bas, E. y Pérez de Guzmán, M. V. (2010). Desafíos de la familia actual ante la escuela y las tecnologías de información y comunicación. Educatio Sig/o XXI, 28(1), 41-68. Recuperado de https://bit.ly/318376z

Cánovas, G., García de Pablo, A., Oliaga, A. y Aboy, I. (2014). Menores de edad y conectividad en España: Tablets y Smartphones. Madrid: Centro de Seguridad en Internet para los Menores en España: Protégeles.

ComScore (2013). 2013 Spain Digital Future in Focus - El Mercado Digital Español. Recuperado de https://cutt.ly/jaLapki

Crescenzi, L. y Grané, M. (2016). Análisis del diseño interactivo de las mejores apps educativas para niños de cero a ocho años. Comunicar: Revista científica iberoamericana de comunicación y educación, 24(46), 77-85. https://doi.org/10.3916/C46-2016-08

Crescenzi, L., Valente, R. y Suárez, R. (2019). Aplicaciones educativas seguras e inclusivas: La protección digital desde una perspectiva ética y crítica. Comunicar: Revista científica iberoamericana de comunicación y educación, 27(61), 93-102. doi: 10.3916/C61-2019-08

Del Moral, M.E. (2004). Pautas procedimentales para el diseño y análisis de videojuegos desde una perspectiva educativa. En M.E., Del Moral (Coord.), Sociedad del Conocimiento, Ocio y Cultura: Un enfoque interdisciplinar (pp. 33-64). Oviedo: Editorial KRK.

Ditrendia (2020). Mobile en España y en el Mundo 2020 + Especial COVID-19. Recuperado de https://bit.ly/37405Lu

Ebner, M., Schönhart, J. y Schön, S. (2014). Experiences with Ipads in Primary School. Profesorado. Revista de currículum y formación del profesorado, 18(3), 161-173. Recuperado de https://bit.ly/2Jf6sDT

González, J.L., Cabrera, M., y Gutiérrez, F. L. (2007). Diseño de Videojuegos aplicados a la Educación Especial. En. J. A. Macías, A. Granollers y P. M. Latorre (Coord.), Actas del VIII Congreso Internacional de Interacción Persona Ordenador (INTERACCIÓN 2007). pp. 35-44

González, J.L., y Gutiérrez, F. L. (2014). Jugabilidad como medida de calidad en el desarrollo de videojuegos. Paper presented at the CoSECivi (Congreso de la Sociedad Española para las Ciencias del Videojuego).

Guernsey, L. (2013). Screen Time: How Electronic Media - From Baby Videos to Educational Software - Affects Your Young Child. New York: Basic Books.

Herodotou, C. (2018). Young children and tablets: A systematic review of effects on learning and development. Journal of Computer Assisted Learning, 34(1), 1-9. https://doi. org/10.1111/jcal.12220

Krippendorff, K. (1990). Metodología del análisis de contenido. Teoría y práctica. Barcelona: Paidós.

López-Gómez, S. (2018). Análise descritiva e interpretativa do deseño e contido dos videoxogos elaborados en Galicia [Tesis de doctorado, Universidade de Santiago de Compostela]. https://bit.ly/2VcOfed

López-Raventós, C. (2016). El videojuego como herramienta educativa. Posibilidades y problemáticas acerca de los serious games. Apertura, 8(1),1-15.

Marín, V. y Martín, J. (2014). Can videogames be used to develop the infant stage educational curriculum? New Approaches in Educational Research, 3(1), 20-25. 
Madrid, D., Mayorga, M. y Núñez, F. (2013). Aplicación del m-learning en el aula de primaria: Experiencia práctica y propuesta de formación para docentes. Edites. Revista Electrónica De Tecnología Educativa, 45, 1-12. https://doi.org/ 10.21556/ edutec.2013.45.27

Mangiron, C. (2011). Accesibilidad a los videojuegos: estado actual y perspectivas futuras. TRANS. Revista de traductología, 15, 53-67. https://doi.org/ 10.24310/TRANS.2011. v0i15.3195

Matsumoto, M., Aliagas, C., Morgade, M., Correro, C., Galera, N., Roncero, C. y Póveda, D. (2016). Young Children (0-8) and Digital Technology. A qualitative exploratory study. Joint Research Centre (JRC) European Commission: UAM y UAB.

Miles, M.B., Huberman, A.M. y Saldaña, J. (2014). Qualitative Data Analysis: A Methods Sourcebook. Los Angeles: Sage.

Moreira, K.D. y Gómez, R.J. (2019). El uso de los equipos tecnológicos y su incidencia en el desarrollo de los procesos atencionales de los niños de cuatro años en la escuela de educación general básica San Francisco de Quito del período lectivo 2018-2019 [Proyecto de investigación, Universidad Laica Vicente Rocafuerte de Guayaquil]. Accesible en https://bit.ly/2KITHSB

Nogueira, M.A. y Ceinos, M.C. (2015). Influencia de la tablet en el desarrollo infantil: perspectivas y recomendaciones a tener en cuenta en la orientación familiar. Tendencias pedagógicas, 26, 33-50.

Pascual, I. (2019). ¿Sociología de la Infancia? Aproximaciones a un campo de estudio difuso. Revista Internacional de Sociología, 58(26), 99-124.

Quiñones, A. (2014). Padres y menores: necesidades formativas ante riesgos y delitos digitales. En M. Reina, A. Navarro, J.L. Monestier y F. J. Durán (Coord.), La sociedad digital: oportunidades y retos para menores y jóvenes (pp. 188-195). Universidad de Granada: Editorial Comares.

Redbility (2013). \#InformeTAB. Estudio sobre el comportamiento de los usuarios de tablet en España. Universidad Internacional de la Rioja.

Rico, M.M. y Agudo, J.E. (2016). Aprendizaje móvil de inglés mediante juegos de espías en Educación Secundaria. RIED (revista iberoamericana de educación a distancia), 19(1), 121-139. http://revistas.uned.es/index.php/ried/article/view/14893/13571

Rodríguez-López, X. (1997). As tarefas académicas e a súa relación cos contidos: análise das actividades docentes no segundo ciclo de Ensino primário, coñecemento de meio, língua galega e matemáticas [Tesis de doctorado no publicada]. Universidade de Santiago de Compostela.

Romero, Y. S., Fernández, A.A., Meneses, E.L., Almenara, J.C. y Gómez, J.I.A. (2012). Las tecnologías de la información en contextos educativos: Nuevos escenarios de aprendizaje. Santiago de Cali: Universidad Santiago de Cali.

Sanchis, V.M. (2019). Aplicaciones digitales para el desarrollo de la lectoescritura para el alumnado de Educación Infantil y Primaria. En R. Roig-Vila (Coord.). Redes de Investigación e Innovación en Docencia Universitaria. Alicante: Universidad de Alicante, Instituto de Ciencias de la Educación (ICE).

Zapico, M.H. (2012). Presenza, conceptualización e tratamento da vellez no currículo escolar: quimera ou realidade? Análise da imaxe das persoas maiores nos materiais curriculares de Educación Primaria de Galicia [Tesis de doctorado no publicada]. Universidade de Santiago de Compostela. 